\title{
Major Incident triage: derivation and comparative analysis of the Modified Physiological Triage Tool (MPTT).
}

\section{Background:}

Triage is a key principle in the effective management at a major incident. There are at least three different triage systems in use worldwide and previous attempts to validate them, have revealed limited sensitivity. Within a civilian adult population, there has been no work to develop an improved system.

\section{Methods:}

A retrospective database review of the UK Joint Theatre Trauma Registry was performed for all adult patients (>18 years) presenting to a deployed Military Treatment Facility between 2006-2013. Patients were defined as Priority One if they had received one or more life-saving interventions from a previously defined list.

Using first recorded hospital physiological data (HR/RR/GCS), binary logistic regression models were used to derive optimum physiological ranges to predict need for life-saving intervention. This allowed for the derivation of the Modified Physiological Triage Tool - MPTT (GCS $<14$, HR $>100$, $12<\mathrm{RR}>22$ ). A comparison of the MPTT and existing triage tools was then performed using sensitivities and specificities with $95 \%$ confidence intervals. Differences in performance were assessed for statistical significance using a McNemar test with Bonferroni correction.

\section{Results:}

Of 6095 patients, $3654(60.0 \%)$ had complete data and were included in the study, with 1738 (47.6\%) identified as priority one. Existing triage tools had a maximum sensitivity of $50.9 \%$ (Modified Military Sieve) and specificity of 98.4\% (Careflight). The MPTT (sensitivity 69.9\%, 95\% CI $0.677-0.720$, specificity $65.3 \%, 95 \%$ CI $0.632-0.675)$ showed an absolute increase in 
sensitivity over existing tools ranging from $19.0 \%$ (Modified Military Sieve) to $45.1 \%$ (Triage Sieve). There was a statistically significant difference between the performance $(p<0.001)$ between the MPTT and the Modified Military Sieve.

\section{Discussion \& Conclusion:}

The performance characteristics of the MPTT exceed existing major incident triage systems, whilst maintaining an appropriate rate of over-triage and minimising under-triage within the context of predicting the need for a life-saving intervention in a military setting. Further work is required to both prospectively validate this system and to identify its performance within a civilian environment, prior to recommending its use in the major incident setting.

\section{Keywords:}

Triage, Major incidents, life-saving intervention, physiological predictors 


\section{Major Incident triage: derivation and comparative analysis of the Modified Physiological Triage Tool (MPTT).}

\section{Introduction:}

Triage is a key principle in the effective management of major incidents and, in line with the principles taught by the Major Incident Medical Management and Support course, is the first clinical priority, ahead of casualty treatment. ${ }^{1}$ Stemming from the French verb trier, meaning to sort, its origins can be traced back to the $14^{\text {th }}$ Century where it was used to describe sorting coffee beans and wool. As a clinical 'sorting' process, Baron Larrey, Napoleon's surgeon, is frequently credited with introducing the first system around 1792; "Those who are dangerously wounded should receive the first attention, without regard to rank or distinction"., 2,3 Today, within the clinical context, it is regarded as the process of "sorting patients and categorising them on the basis of clinical acuity". 4

Triage is a dynamic process, and at a major incident it will be repeated several times as the patient transitions through the respective phases of medical care. ${ }^{1}$ Equally, the physiological state of the patient may improve following intervention, or deteriorate in response to injury progression; having a dynamic triage process, where the patient can undergo repeated assessment, allows for this to be recognised and the patient category can be amended as required. A key tenet of initial major incident triage is that it can be performed rapidly, and the results should be reproducible irrespective of user.

A number of triage methods exist (Triage Sieve, START, Careflight), ${ }^{1,5}$ each assigning patients to one of three priority categories. The initial discriminator of these tools is the ability to walk; 
patients able to walk are allocated the lowest acuity category (P3 or delayed). Those unable to walk then undergo an assessment of basic physiology (Heart Rate, Respiratory Rate, Blood Pressure and Conscious Level) to assign patients to either P1 or P2 categories (immediate or urgent). ${ }^{1,5}$ Although mechanism of injury and anatomical injury are used for individual field triage, within a major incident setting, these are too time-consuming and require additional user training. ${ }^{6}$

The most severely injured patients (priority one) are those requiring life-saving interventions, the definition of which has evolved over time since it was first used for individual trauma patients. ${ }^{5,7}$ Definitions exist for the paediatric and military patient, but until recently there has not been an accepted, consensus definition of what constitutes a civilian priority one adult patient at a major incident. $^{4,8,9}$

To date, there has been no prospective validation of the major incident triage tools during a major incident; this is unlikely to be possible in the future due to both ethical and logistical constraints. Research is therefore limited to either the retrospective review of major incidents or the analysis of trauma registry data. There have been a limited number of reviews comparing the performance of existing triage tools.

An early trauma registry review demonstrated similar performance of START and Careflight (sensitivity $84 \%$ and $82 \%$, specificity $91 \%$ and $86 \%$ respectively) with the Triage Sieve performing poorly at predicting the need for life-saving intervention (sensitivity $45 \%$, specificity $88 \%$ ). ${ }^{5}$ By contrast, a retrospective comparison of the same triage tools following the London $7^{\text {th }}$ July bombings demonstrated equally poor sensitivities (50\%) for all triage tools, albeit all with $100 \%$ specificity. ${ }^{10}$ However, despite using the same life-saving intervention definition and it being a major incident, only $2 \%(n=4)$ patients included were considered priority one, in contrast to a registry review with $12 \%(\mathrm{n}=135) .{ }^{5}$ With only a single study validating modifications to an existing 
triage tool, the Modified Military Sieve, there has been no work to date to derive an optimum physiological triage tool. ${ }^{11}$

With lack of evidence to support existing major incident triage tools, this study aims to derive a triage tool, using observed physiological measurements, that shows an improved performance at predicting the need for life-saving intervention in a military population when compared to existing methods.

\section{Methods:}

A retrospective database review of the UK Joint Theatre Trauma Registry was conducted for all adult trauma patients ( $\geq 18$ years) presenting to the Emergency Department at Camp Bastion, Afghanistan between 2006-2013. The medical facilities provided at Camp Bastion have been extensively described elsewhere. ${ }^{12,13}$

Data on all seriously injured patients (including UK military, coalition forces, detainees, and local civilians) treated by UK Defence Medical Services in these facilities are collected by Trauma Nurse Coordinators within the deployed clinical team and returned to the UK Joint Theatre Trauma Registry (JTTR). Defence Analytical Services and Advice maintain the JTTR at the Royal Centre for Defence Medicine in Birmingham, UK. Data are collected from clinical notes, trauma charts and in the case of death, post mortem findings. The JTTR holds continuous data on this cohort from 2003, coinciding with the start of hostilities in Iraq. Returns are electronic (where deployed IT systems allow), with hard copy accompanying UK military patients evacuated to Royal Centre for Defence Medicine for definitive care. ${ }^{14}$ 
The default entry criterion for UK JTTR is a casualty who triggers trauma team activation in a deployed field hospital or Primary Casualty Receiving Facility afloat. The entry criteria were expanded in 2007 to include all trauma patients returned to Royal Centre for Defence Medicine for definitive treatment, irrespective of whether a trauma team response was mandated. Anonymised data were supplied from the JTTR database, and according to institutional agreement ethical approval was not required. ${ }^{14}$

Only patients with complete recordings of their physiological parameters on arrival at hospital were included in the study (SBP, HR, GCS, RR). Due to the nature of the JTTR and its inclusion criteria, patients in the study were assumed to be non-ambulant. In order to examine for potential selection bias through the deletion of incomplete records, analysis was performed for age, gender and mechanism of injury for the included and excluded groups. Outliers were defined as a physiological parameter with a $\mathrm{Z}$ score of 3 standard deviations (HR > 170 beats per minute, $\mathrm{SBP}>206 \mathrm{mmHg}$ and $\mathrm{RR}>45$ breaths per minute). In order to prevent bias and reducing statistical power, outliers were removed prior to the analysis. ${ }^{15}$ Patients were defined as priority one if they received any one intervention from a previously derived list (Figure 1). The JTTR does not record presence of a radial pulse as a variable, therefore for the purposes of prioritisation using START and Careflight, a surrogate systolic blood pressure of $90 \mathrm{mmHg}$ was taken to represent the presence of a radial pulse and absence of hypotension. ${ }^{16,17}$

\section{Table 1: List of Life-saving interventions ${ }^{4}$}

The primary outcome of the study was to derive the optimum ranges of each physiological parameter in isolation at predicting the need for life-saving intervention. The secondary outcome was to compare the performance of the Modified Physiological Triage Tool (MPTT), the combination of these independently derived parameters, with existing major incident triage tools. 
Separate bivariate logistical regression models were developed for each physiological parameter in isolation to determine the need for life-saving intervention. For heart rate and respiratory rate, regressions were estimated separately for values above and below the median (HR-89, RR-18). The performance of each model was reported in terms of the significance of the parameters (B0 and B1), the explanatory power (Nagelkerke's pseudo-R2) and goodness of fit (Hosmer-Lemeshow's $\chi^{2}$ ). The probability of outcome equation Probability (event $\mathrm{Y})=1 /\left(1+\mathrm{e}^{-(\mathrm{B} 0+\mathrm{X} * \mathrm{~B} 1)}\right)$ was used to determine the optimum threshold for predicting need for life-saving intervention for each physiological parameter in isolation.

For the comparative analysis, performance was evaluated using sensitivity, specificity, under-triage (1-sensitivity) and over-triage (1-Positive Predictive Value) with 95\% confidence intervals calculated for all major incident triage tools. For tools with similar performance, a McNemar test with a Bonferroni correction was applied, allowing for the evaluation of any statistically significant difference between the tools. ${ }^{18}$ Data processing and analysis were conducted using a combination of Statistical Package for the Social Sciences (SPSS) Version 23.0 (SPSS Inc., Chicago, Illinois, USA), STATA Version 12.0 (StataCorp, College Station, Texas, USA) and Microsoft Excel Version 14.5.8. ${ }^{19-20}$

As part of a larger programme of work, this study received ethical approval by the Human Research Ethics Committee of the University of Cape Town (reference 285/2013), the primary institution of the lead author.

\section{Results:}


During the study period 6095 adult patients were included in the database. $3701(60.7 \%)$ had complete physiological data (SBP, HR, GCS and RR) with 47 excluded as outliers (SBP > $206 \mathrm{n}=9$, $R R>45 n=28, H R>170, n=12) .3654$ were included in the final data analysis $(60.0 \%)$ with a median age of 24 years (IQR 21-29 years); 3593 (98.3\%) were male. Both independent t-test and Pearson Chi Square tests were non significant for age $(\mathrm{p}=0.811)$ and gender $(\mathrm{p}=0.472)$ respectively when comparing the complete and incomplete physiological data groups. Statistical significance was observed for mechanism of injury between the two groups $(\mathrm{p}<0.05)$; however, observationally the relative frequencies were similar for both explosive (57.5\% vs $55.1 \%)$ and GSW (30.6\% vs $34.3 \%$ ) mechanisms of injury.

During the study period there were $75(2.1 \%)$ fatalities. Injury secondary to explosive devices and gunshot wounds combined accounted for the majority of cases $(n=3264,89.3 \%)$. Injured personnel had a mean of 2 body regions affected (range 0-8) with the highest proportion affecting the lower extremities $(36.0 \%)$, followed by upper extremities (16.2\%) and thorax (10.8\%). Injury Severity Score (ISS) was recorded for the majority of patients $(n=3649,99.8 \%)$, with median and mean ISS of 5 and 11.4 respectively.

$1738(47.6 \%)$ patients received a life-saving intervention and were considered Priority One, with the majority receiving a single intervention $(n=629,36.2 \%)$, range 0 to 12.5380 life-saving interventions were performed during the study period with tourniquet use the most frequent $(n=724$, 13.5\%). No patients received chemical antidotes, therapeutic rewarming or correction of low blood glucose. Additionally, no patients received interventional radiology for haemorrhage control as it is not currently a deployed medical capability of the UK Defence Medical Services.

Table 2: Characteristics of study population 


\section{Glasgow Coma Scale}

The regression model demonstrated significance $\left(\mathrm{p}<0.001, \chi^{2}=768.42\right)$, explaining approximately $25 \%$ of the variation in the outcome variable (Nagelkerke $\left.\mathrm{R}^{2}=0.255\right)$. The model fit was satisfactory (Hosmer and Lemeshow statistic $\chi^{2}=0.441, \mathrm{df}=2, \mathrm{p}=0.506$ ). Using a probability of outcome equation, the value of GCS $<14$ was derived as the optimum level for predicting the need for life-saving intervention. Probability values for all physiological parameters are provided in tabulated form in Tables 1-3, web only appendices.

\section{Figure 1: GCS Parameter Estimates}

\section{$\underline{\text { Respiratory Rate }}$}

Both regression models $(\mathrm{RR} \leq 18$ and $\mathrm{RR}>18)$ demonstrated significance, $\chi^{2}=21.4$ and 75.2 , d.f $=1$, $\mathrm{p}<0.001$ respectively, with poor fit (Hosmer and Lemeshow statistic $\chi^{2}=27.8$ and 13.5, d.f $=6$ and $\mathrm{p}<0.05$ respectively for $\mathrm{RR} \leq 18$ and $\mathrm{RR}>18$ ). Optimum levels of respiratory rate (upper and lower) were defined as $\mathrm{RR}<12$ and $\mathrm{RR} \geq 22$ having been derived using probability of outcome equations. (Figures $1 \& 2$, web only appendices)

\section{$\underline{\text { Heart Rate }}$}

Only the HR $>89$ model demonstrated significance, $\chi^{2}=179.6$, d.f $=1$ and $p<0.001$ with a good fit (Hosmer and Lemeshow statistic $\chi^{2}=8.8$, d.f. 8 and $\mathrm{p}=0.358$ ). Using the probability of outcome equation, a HR threshold of $\geq 100$ was determined as the optimum level at predicting need for lifesaving intervention. (Figure 3, web only appendix)

\section{$\underline{\text { Comparative Analysis }}$}


The MPTT, defined as GCS $<14, \mathrm{RR}<12, \mathrm{RR} \geq 22, \mathrm{HR} \geq 100$, demonstrated the greatest sensitivity of all existing triage tools $(69.9 \%, 95 \%$ CI $67.7-72.0 \%)$, with an absolute increase in sensitivity of $19.0 \%$ over the Modified Military Sieve and the lowest rate of under-triage (30.1\%). Statistically significant differences were recorded between both the MPTT and Modified Military Sieve $\left(\chi^{2}=746, \mathrm{p}<0.001\right)$ and the MPTT and Military Sieve $\left(\chi^{2}=998, \mathrm{p}<0.001\right)$. Figure 3 summarises the performance accuracy of the triage tools in their ability to predict the need for lifesaving intervention.

\section{Figure 2: MPTT Flowchart}

\section{Figure 3: Triage tool performance}

Table 3: Sensitivity analysis.

\section{Discussion:}

This study has successfully derived the first evidence-based triage tool with improved sensitivity and acceptable specificity when compared to existing tools such as START, Careflight and the Military Sieve. This was achieved using a cohort of military patients.

There is a paucity of literature surrounding both the creation and use of existing major incident triage tools. No previous study has attempted to derive a tool based on physiological data. Having adapted the criteria defining a major trauma patient to reflect major incident practice, one study performed a comparative analysis to demonstrate the performance of existing tools and using logistical regression methods, identified the strengths and weaknesses of the current thresholds for each physiological component. ${ }^{5}$ The performance of both START and Careflight differed largely from that observed subsequently; ${ }^{9,21}$ the use of a lower SBP surrogate to represent palpable radial pulse $(80 \mathrm{mmHg})$ may explain some of the differences in sensitivity observed. ${ }^{5,10,11}$ 
Following the $7^{\text {th }}$ July bombings in London, all tools (START, Triage Sieve and Careflight) were shown to have the same performance at identifying priority one patients (50\% sensitivity and $100 \%$ specificity). ${ }^{21}$ Despite being performed on a major incident dataset, data were only available for $50 \%$ of the small number of priority one patients, from one hospital $(n=4)$. Additionally, a SBP of $110 \mathrm{mmHg}$ was used represent the presence of a palpable pulse when categorising patients using START and Careflight. Although a wide range of systolic blood pressures correlating with palpable pulse have been used in previous studies, this estimate is higher than most and a surrogate of $90 \mathrm{mmHg}$ as was used in this study, may be more appropriate with its correlation with increased mortality following both penetrating and blunt trauma. ${ }^{22,23}$

Within a military setting, simple modifications have been proposed to the heart rate and respiratory rate components of the military sieve, demonstrating an improvement in its performance characteristics. ${ }^{9}$ During a subsequent prospective validation and comparative analysis, the modified military sieve was demonstrated to have a statistically significant increase in performance when compared to existing methods. ${ }^{11}$

\section{Table 4: Comparative analysis by study., 5,10}

This study has successfully created the Modified Physiological Triage Tool and is the first study where physiological thresholds within triage tools have been derived using logistical regression to individually predict need for life-saving intervention. Whilst ISS was measured within our population, we chose specifically to measure triage tool performance against need for life-saving intervention. Numerous studies have previously demonstrated a lack of correlation between ISS and need for life-saving intervention. Fundamentally, the ISS is a retrospectively calculated score which measures injury severity and does not describe the clinical acuity of the patient. The high 
frequency of $\mathrm{P} 1$ patients (47.6\%) reflects the injury burden within our cohort. Within a major incident setting the authors believe that this is a more appropriate measure. ${ }^{4,7,8,10}$

The MPTT showed the greatest sensitivity $(69.9 \%, 95 \%$ CI $67.7-72.0 \%)$ at predicting the need for life-saving intervention. With the lowest rate of under-triage, the MPTT demonstrates far better performance clinically and statistically than existing tools, with an absolute increase in sensitivity of $19.0 \%$ over the Modified Military Sieve (50.9\%, 95\% CI 48.6-53.3\%). However, this increased sensitivity comes with a reduction in specificity and the highest rate of over-triage (35.2\%).

Currently there is no guidance to stipulate the recommended accuracy of major incident triage, however for field triage to a Major Trauma Centre, the recommendations are that over and undertriage are limited to $35 \%$ and $5 \%$ respectively. ${ }^{24}$ The rate of under-triage by the modified physiological triage tool is clearly high, but it is the lowest of all existing major incident triage tools, maintaining a tolerated level of over-triage.

The effect sizes of the individual components of the modified physiological triage tool are in themselves small, with only a maximum of $13 \%$ variation accounted for by both HR and GCS (Nagelkerke's $\mathrm{R}^{2}$ ). With the nature of the modified physiological triage tool's derivation, the performance we have demonstrated is likely to represent the optimum for simple physiological parameters as is contained within major incident triage tools. Without including additional measured variables (such as mechanism of injury or anatomical injury), these rates of over and under-triage are unlikely to be improved, as this would render the triage tool unsuitable for the 'quick-look' primary triage that is required initially at the scene of a major incident.

A key principle of major incident triage is that it must be able to be performed rapidly, reliably and with reproducible results, irrespective of the seniority or background of the individual performing it. 
The modified physiological triage tool has been derived with these principles in mind and is no more complicated than existing tools, yet demonstrates increased performance at predicting the need for life-saving intervention, whilst minimising rates of under-triage and having an acceptable rate of over-triage.

\section{Limitations:}

There are a number of limitations to our study, the first being the use of a military trauma registry to derive a major incident triage tool.

Patients who were uninjured or sustained minor injuries following an incident are not included in the JTTR. Specificities within the analysis must therefore be interpreted with caution as the inclusion criteria will prevent all 'true negative' patients from being included within the JTTR. The population of patients from a major incident population will include a large number of patients sustaining only minor injuries, representing the ambulant P3 or delayed category. Whilst trauma registries have been and continue to be used as surrogates for major incident research, they are unlikely to be entirely representative of the population in question because of this, instead focusing on the non-ambulant patients of higher acuity. ${ }^{5}$

Our dataset demonstrates a high proportion of priority one patients ( $\mathrm{n}=1755,47.8 \%)$, most of whom suffered blast and ballistic injury $(\mathrm{n}=3268,89.0 \%) .{ }^{25}$ Over the last decade several high profile terrorist atrocities have occurred across Europe, including a number of marauding terrorist firearm attacks, using high velocity weapons and improvised explosive devices akin to that seen in the military setting. ${ }^{26}$ Whilst the MPTT has demonstrated good performance at predicting need for lifesaving intervention within our cohort, and may be likely to do so in the context of terrorist atrocities 
on civilians, its performance may not easily translate to a civilian major incident if the mechanism of injury is predominantly blunt trauma. ${ }^{25}$

Closely linked with the mechanism of injury experienced on the battlefield is the relatively low age (median 24 years, mean 26.2 years) and the low frequency of females injured within our dataset $(n=60,1.6 \%)$. Within a UK civilian trauma context it has been acknowledged that the mean age of patients has increased over the last three decades and in 2013 was 53.8 years. ${ }^{25}$ We also recognise that our cohort of predominantly young males are likely to have limited medical comorbidities when compared to the population as a whole, and therefore the physiology with which we have derived the MPTT is likely to not be fully representative of the whole population. Whilst this is not specific to the major incident environment, it is likely that it will be relevant and these factors are acknowledged as limitations to our study and must be explored before the MPTT can be recommended for use in a civilian context.

Evaluating the performance of the MPTT on the same dataset in which it was derived introduces the potential for bias with respect to its performance. It has previously been suggested that in these circumstances, the derived diagnostic test (in this case the MPTT) can have an exaggerated test performance and that when evaluated using an alternative, independent dataset performs less well. ${ }^{27}$ Analysis using additional independent datasets are therefore required to support the MPTT's improved performance.

Whilst our analysis has been performed on a large sample $(n=3673)$, it is acknowledged that a number of patients were removed due to incomplete physiological data $(n=2394,39.3 \%$ of total dataset). The list-wise deletion method of incomplete data can be considered as a form of selection bias, but analysis on the deleted dataset revealed no statistically significant difference between age and gender of the two groups. Although significance was seen with mechanism of injury between 
the two groups, observationally the proportions were near identical. Despite our results demonstrating both considerable effect sizes and significance, we are unable to comment whether with a more complete data set these results would have been different. Incomplete data entry is a limitation of retrospective database reviews, and the JTTR is no different. The nature of military operations and the pressured environment that clinicians are working in, is likely to explain some of this missing data. ${ }^{11}$ The major incident setting is no different, and the difficulties in maintaining contemporaneous medical records during a major incident have been described previously. ${ }^{21,28}$ The extent of missing data in our study (39.3\%) is directly comparable to that observed following the $7^{\text {th }}$ July bombings (approximately $38.0 \%$ ).

We acknowledge that the use of in-hospital physiology is a limitation of our study. A change in physiology may well be observed in patients receiving interventions prior to their arrival at hospital. Whilst data is available on the JTTR at point of wounding, only $25 \%$ cases for the study period in question had complete data. Due to the austere nature of military operations, data completion is unsurprisingly poor, and this has previously been described elsewhere. ${ }^{29}$ For this practical reason physiology was used on arrival at hospital, where complete data was available for $61 \%$.

In keeping with the literature on mortality following trauma, a surrogate systolic blood pressure measurement of $90 \mathrm{mmHg}$ was used to represent the presence of a radial pulse for purposes of classification using START and Careflight. ${ }^{22,23}$ We acknowledge that the use of surrogates is a limitation of our study, but one that is shared with other major incident triage publications and due to the nature of the JTTR is unavoidable..$^{5,10,30}$

Table 5: Surrogates used within the study

\section{Conclusions:}


In summary, we present the modified physiological triage tool, the first example of a statistically derived physiological triage tool for use in the military major incident setting. Our findings show that the modified physiological triage tool demonstrates good performance at predicting need for life-saving intervention within a military setting. It is superior to all existing major incident triage tools with respect to its rates of under-triage, and has an acceptable level of over-triage. Further work is needed to validate this tool on civilian trauma registry data and will be required prior to changes to existing civilian major incident doctrine. Ideally, the modified physiological triage tool should be specifically tested in the major incident environment in order to assess its performance. 


\section{References:}

1. Advanced Life Support Group. Major Incident Medical Management and Support: The Practical Approach. London: BMJ Books, 2002

2. Trauma.org. United Kingdom. Cited 30 May 2016. Available on http://www.trauma.org

3. Burris D, Welling D \& Rich N. Dominique Jean Larrey and the Principles of Humanity in Warfare. J Am Col Surg 2004; 198 (5): 831-835

4. Vassallo J, Smith J E, Bruijns S, Wallis L A. Major incident triage: a consensus based definition of the essential life-saving interventions during the definitive care phase of a major incident. Injury 2016; 47 (9):1898-1902

5. Garner A, Lee A, Harrison K, Schultz C. Comparative Analysis of Multiple-Casualty Incident Triage Algorithms. Ann Emerg Med 2001; 38 (5): 541-548

6. Sasser S, Hunt R, Faul M et al. Guidelines for Field Triage of Injured Patients. Recommendations of the National Expert Panel on Field Triage. MMWR Recomm Rep. 2009;58(RR-1):1-35

7. Baxt WG \& Upenieks V. The lack of full correlation between the injury severity score and the resource needs of injured patients. Ann Emerg Med 1990; 19: 1396-1400

8. Wallis L, Carley S \& Hodgetts C T. A procedure based alternative to the injury severity score for major incident triage of children: results of a Delphi consensus process. Emerg Med J 2006; 23(4):291-5

9. Horne S, Vassallo J \& Read J. UK Triage - a new tool for an evolving threat. Injury 2013; 44 (1): $23-28$

10. Challen K H E (2008) Comparative validation of major incident triage systems. MRes thesis. University of Manchester. 
11. Vassallo J, Horne S, Ball S, Whitley J. UK Triage - the validation of a new tool for an evolving threat. Injury 2014; 45 (12):2071-5

12. Henning D, Smith J E, Patch D, Lambert A. A comparison of civilian (National Confidential Enquiry into Patient Outcome and Death) trauma standards with current practice in a deployed field hospital in Afghanistan. Emerg Med J 2011; 28 (4): 310-312

13. Vassallo D. A short history of Camp Bastion Hospital: the two hospitals and unit deployments. J R Army Med Corps 2015;161:79-83.

14. Smith JE. The epidemiology of blast lung injury during recent military conflicts: a retrospective database review of cases presenting to deployed military hospitals, 2003-2009. Phil. Trans. R. Soc. B. 2011; 366: 291-294

15. Field A. Discover statistics using SPSS. $3^{\text {rd }}$ edn. London: SAGE publications, 2009.

16. Edelman, D. A., White, M. T., Tyburski, J. G, Wilson R F. Post-traumatic hypotension: should systolic blood pressure of 90-109 mmHg be included? Shock 2007; 27(2): 134-138

17. Rossaint R, Bouillon B, Cerny V et al. Management of bleeding following major trauma: an updated European guideline. Crit Care 2010;14:R52.

18. Hazra A, Gogtay N. Biostatistics series module 4: Comparing groups - categorical variables. Indian J Dermatol 2016; 61:385-92

19. IBM Corp. Released 2013. IBM SPSS Statistics for Macintosh, Version 23.0. Armonk, NY:IBM Corp.

20. StataCorp. 2011. Stata Statistical Software: Release 12. College Station, TX: StataCorp LP.

21. Challen K, Walter D. Major Incident Triage: Comparative validation using data from $7^{\text {th }}$ July bombings. Injury 2013;44(5):629-633

22. Hasler, R. M., Nüesch, E., Jüni, P, Bouamra O, Exadaktylos A, Lecky F. Systolic blood pressure below $110 \mathrm{mmHg}$ is associated with increased mortality in penetrating major trauma patients: Multicentre cohort study. Resuscitation, 2012; 83(4): 476-481 
23. Hasler, R. M., Nüesch, E., Jüni, P, Bouamra O, Exadaktylos A, Lecky F. Systolic blood pressure below $110 \mathrm{mmHg}$ is associated with increased mortality in blunt major trauma patients: Multicentre cohort study. Resuscitation, 2011; 82(9): 1202-1207

24. American College of Surgeons. Resources for the optimal care of the injured patient: 2014. Chicago, IL: American College of Surgeons; 2014.

25. Kehoe A, Smith JE, Edwards A, Yates D, Lecky F. The changing face of major trauma in the UK. Emerg Med J 2015;32:911-915

26. Hirsch M, Carli P, Nizard R, Riou B, Baroudjian B, Baubet T, Chhor V, Chollet-Xemard C, Dantchev N, Fleury N, Fontaine JP, Yordanov Y, Raphael M, Paugham Burtz C, Lafont A. The medical response to multisite terrorist attacks in Paris. Lancet 2015; 386(10012):25352538 .

27. University of York 2009. Diagnostic Tests. In Systematic Reviews. Available on: https://www.york.ac.uk/crd/SysRev/!SSL!/WebHelp/TITLEPAGE.htm. Cited $9^{\text {th }}$ January 2017.

28. London Assembly. Report of the $7^{\text {th }}$ July Review Committee. June 2006

29. Robinson J B, Smith M P, Gross K R, Sauer S W, Geracci J J, Day C D, Kotwal R S. Battlefield documentation of tactical combat casualty care in Afghanistan. US Army Med Dep J 2016; (2-16):87-94

30. Price C L, Brace-McDonnell S J, Stallard N, Bleetman A, Maconochie I, Perkins G D. Performance characteristics of five triage tools for major incidents involving traumatic injuries to children. Injury 2016; 47:988-992 
